\title{
Lateral neck cysts, age distribution and diagnostic pathway
}

\author{
Rishi Shukla ${ }^{1}$, Ketan Shah ${ }^{2}$, and stuart winter ${ }^{2}$ \\ ${ }^{1}$ Oxford University Hospitals NHS Foundation Trust \\ ${ }^{2}$ John Radcliffe Hospital
}

September 21, 2020

\begin{abstract}
Objectives The objectives of this study are to describe the incidence and age range of presentation of lateral neck cysts, to evaluate a management algorithm for adults presenting with lateral neck cystic lesions and to report the age incidence at which malignant cystic lesions in the neck have clinically presented as lateral neck cysts. Design A retrospective review of histologically diagnosed branchial cysts between 1995 and 2014 at a single centre. Setting Oxford University Hospitals NHS Foundation Trust. Participants A single senior head and neck pathologist and the ENT head and neck team. Main outcome measures Does a dedicated pathway help to identify malignancy in lateral neck cysts earlier in the diagnostic pathway? Results When using a random investigative pathway the sensitivity in distinguishing malignant from non-malignant was $93 \%$, specificity $12 \%$ with a PPV of $83 \%$ and NPV $28 \%$. When using a defined investigative protocol, the sensitivity was $94 \%$, specificity $67 \%$, PPV of $85 \%$ and NPV $86 \%$. Conclusions This study conveys that using a structed pathway when working up patients with a lateral neck cyst results in a greater sensitivity, specificity, PPV and NPV in distinguishing malignancy compared to an unstructured workup. Key points 1) Establishing a lateral neck cyst as a benign structure is a diagnosis of exclusion 2) There is no established protocol for this pathway. 3) The need for a diagnostic pathway is important given the impact of COVID-19 4) The diagnostic challenge is differentiating benign from malignant 5) For lateral neck cysts a cut off age of 35 may not be appropriate
\end{abstract}

\section{Abstract: Objectives}

The objectives of this study are to describe the incidence and age range of presentation of lateral neck cysts, to evaluate a management algorithm for adults presenting with lateral neck cystic lesions and to report the age incidence at which malignant cystic lesions in the neck have clinically presented as lateral neck cysts.

Design

A retrospective review of histologically diagnosed branchial cysts between 1995 and 2014 at a single centre.

Setting

XXX University Hospitals NHS Foundation Trust.

Participants

A single senior head and neck pathologist and the ENT head and neck team.

Main outcome measures

Does a dedicated pathway help to identify malignancy in lateral neck cysts earlier in the diagnostic pathway?

Results

When using a random investigative pathway the sensitivity in distinguishing malignant from non-malignant was $93 \%$, specificity $12 \%$ with a PPV of $83 \%$ and NPV $28 \%$. When using a defined investigative protocol, the sensitivity was $94 \%$, specificity $67 \%$, PPV of $85 \%$ and NPV $86 \%$. 


\section{Conclusions}

This study conveys that using a structed pathway when working up patients with a lateral neck cyst results in a greater sensitivity, specificity, PPV and NPV in distinguishing malignancy compared to an unstructured workup.

Key points

1) Establishing a lateral neck cyst as a benign structure is a diagnosis of exclusion

2) There is no established protocol for this pathway.

3) The need for a diagnostic pathway is important given the impact of COVID-19

4) The diagnostic challenge is differentiating benign from malignant

5) For lateral neck cysts a cut off age of 35 may not be appropriate

Introduction

Benign lateral neck cysts are recognised to present most commonly in children and young adults with the majority classified as branchial cleft cysts ${ }^{1}$. While recognised to occur in adults, little is known about their incidence. In adults, the diagnostic challenge is to differentiate benign lateral neck cysts from other pathology, commonlycystic nodal metastasis of squamous cell carcinoma ${ }^{2}$.

Head and neck cancers (HNC) represent the sixth most common cancer worldwide with approximately 630,000 new patients diagnosed annually. The incidence of HNC has increased significantly over the last 30 years and is this rise is predicted to continue. In part this is due to a significant increase in oropharyngeal cancers associated with the human papilloma virus (HPV). HPV associated squamous cell carcinoma commonly presents with cystic neck metastasis. For this reason, many protocols mandate that above the age of 35 years, a cystic mass is considered malignant until proven otherwise ${ }^{3}$. However, with the increasing incidence of HNC associated with HPV, a cut off age of 35 may not be appropriate ${ }^{4}$.

Establishing a lateral neck cyst as a benign structure is a diagnosis of exclusion, but there is no established protocol for this pathway ${ }^{5}$. The need for a diagnostic pathway has never been more prescient given the impact of COVID-19 and the deferral of a high number of elective operations.

The aims of this study are to describe the incidence and age range of presentation of lateral neck cysts, to evaluate a management algorithm for adults presenting with lateral neck cystic lesions and to report the age incidence at which malignant cystic lesions in the neck have clinically presented as lateral neck cysts.

Patients and Methodology

A retrospective review of histologically diagnosed branchial cysts between 1995 and 2014 at a single centre (XXX University Hospitals NHS Foundation Trust) was undertaken to establish the incidence at different ages. This data was reviewed by a single senior head and neck pathologist.

A retrospective review into management of patients presenting through the XXX head and neck cancer MDT with lateral neck cystic between 2012-2019 was undertaken. Data collected included all diagnostic investigations and the final diagnosis. Patients with clinically apparent primary malignancy, history of head and neck cancer or irradiation, and incomplete radiological and/or cytological workup were excluded from the study.

Patients were stratified into two pathways, an ad-hoc diagnostic pathway with a decision to treat based on the available test results. The other pathway was a defined protocol based on age of presentation. Sensitivity, specificity, positive predictive and negative predictive values for the pathways were calculated.

Results 
Between 1995 and 2018, 205 patients with histologically confirmed branchial cysts were identified. These cases ranged in age from 11 months to 88 years.

Most branchial cysts were noted in patients between the ages of 26 and 40 years, with peak incidence between age 31-35 years (Figure 1). A total of 87 branchial cysts were identified over the age of 35, representing $42 \%$ of the overall cohort.

Between 2012-2019, 102 patients presented to the XX Head and Neck team with a lateral neck cyst; for 5 patients follow up records were not available, and these were excluded. Of the remaining 97, there were 50 male and 47 female patients with an age range of 0-77 years and a mean age of 30 years. The diagnostic pathway was reviewed for this cohort.

During this period some patients were referred having completed an ad-hoc set of investigations, which included ultrasound and/or MRI imaging and fine needle aspiration cytology. Decision recommendations were based on the best available evidence.

For those patients over 35 referred early in their pathway they followed a pre-agreed diagnostic pathway. Figure 2. This involved an incremental number of investigations; clinical examination, USS guided FNAC, PET-CT, surgical excision. If any of these investigations indicated malignancy, then the patient was transferred to an appropriate care pathway.

For those patients investigated under the ad-hoc pathway 71 patients were identified, 35 males and 36 females, with a. mean age of 38 (range 18-75).

Following a review of the available investigations 50 patients had a pre-operative diagnosis of a branchial cyst or other benign lateral neck cyst that was subsequently confirmed based on final histology. 14 patients had a pre-operative diagnosis of branchial cyst or other benign lateral neck cyst but at final histology were confirmed to have a diagnosis of metastatic SCC or other malignant lateral neck cyst. 5 patients had a preoperative diagnosis of highly probable metastatic SCC or other cystic malignant lesion but on final histological assessment had a benign branchial cyst or other benign lateral neck cyst. 2 patients had a pre-operative diagnosis of metastatic SCC or another malignant cystic lesion and this was confirmed histologically. The results for this combined (clinical/imaging/cytology) pathway indicated that the sensitivity 93\%, specificity $12 \%$ with a PPV of $83 \%$ and NPV of $28 \%$.

27 patients were identified having been referred with a cystic lesion in the neck and followed the defined pathway, Figure 2. 15 males and 12 females with a mean age of 58 (range 34-82).

Following a review of the available investigations 17 patients had a pre-operative diagnosis of a branchial cyst or other benign lateral neck cyst that was subsequently confirmed based on final histology. 3 patients were identified with a pre-operative diagnosis of branchial cyst or other benign lateral neck cyst that at final histology was metastatic SCC or other malignant lateral neck cyst. 1 patient had a presumptive diagnosis of a malignant cystic lesion in the neck that on final histology was a reported as a branchial cyst or other benign lateral neck cyst. 6 patients were identified with a pre-operative diagnosis of metastatic SCC or another malignant cystic lesion and this was confirmed on final histology. Using this investigative pathway, the sensitivity was $94 \%$, specificity $67 \%$, PPV of $85 \%$ and NPV of $86 \%$.

Of the 97 patients in this cohort 25 had a final diagnosis of metastatic squamous cell carcinoma. The age distribution is shown in Figure 3. 1 patient (6\%) was under the age of 35. Of those under the age of 35 the age presentation of malignant cystic metastasis was at 21 years old.

Discussion

There remains some debate regarding the cause for benign lateral neck cysts. They are recognised to be a common a common presentation in children and young adults ${ }^{3}$. This study found that $58 \%$ of histologically confirmed branchial cysts occurred in patients under the age of 35 . While the incidence deceased with increasing age, this study found that $42 \%$ of branchial cysts occurred over the age of 35 , with the oldest patient being 78 years old. The most frequent age bracket for occurrence of branchial cysts was 31-35 years. 
While in children a lateral neck cyst is almost certainly benign in nature, in adults the important differentiation is from cystic metastasis,and in particular metastatic HPV associated oropharyngeal carcinoma ${ }^{6}$. This has become an important consideration due to the increasing incidence of oropharyngeal cancer.

There are no convincing cases documenting origin of squamous cell carcinoma from branchial cysts and another possibility is of an unknown primary in the upper aerodigestive tract $^{6}$. In adults, the diagnosis of benign branchial cysts and exclusion of malignancy requiresimaging and cytology, though ultimately histology from an excision biopsy may be needed ${ }^{7}$.

The ability to have a diagnosis of a malignant or benign lesion as early as possible has many advantages. Avoiding an excision biopsy of a malignant cystic lesion in the neck allow for appropriate planning and treatment of malignancy. This study indicates an $8 \%$ prevalence of malignancy in a lateral neck cyst. Other studies have reported a rate of malignancy between 9 to $14 \%$ with malignant tumours found more frequent in patients older than 40 years of age ${ }^{7}$. The disparity in findings in this study may be a reflection of a more aggressive diagnostic pathway or potentially earlier referrals for neck masses in all younger age groups which are subsequently excised before malignant transformation can potentially occur. The diagnostic accuracy of preoperative fine needle aspiration for cytology has been reported to have a sensitivity of $88.8 \%$ and a specificity of $60.0 \%^{2}$. It is the authors opinion that a dedicated head and neck cytopathologist linked into a multidisciplinary review of the history and diagnostic imaging is an advantage.

This study aimed to compare two pathways that have been utilised. It suggests that there is an advantage in having a defined pathway. In particular being able to increase the specificity and negative predictive value of the testing. This has important implications in particular following on from the recent Covid-19 pandemic. During this time procedures on benign lesions have been deferred due to the peri-operative risk of Covid-19 infection and reduced recourses. A failure to recognised malignancy in this period could result in a delay in treatment and a worse prognosis.

The age at which malignancy is actively screened for is frequently quoted as 35 years. This study shows that $6 \%$ percentage of malignancies were detected in under $35 \mathrm{~s}$. This suggests that a cut off of 35 may not be appropriate and that this could be lowered to 20 which would cover $6 \%$ of patients

These findings need to be interpreted due to the limitations of the study design; retrospective and a small cohort.

\section{References}

1. Franzen et al. (2019). Cystic Lateral Neck Lesions: Etiologic and Differential Diagnostic Significance in a Series of 133 Patients. Anticancer Research, 39(9), 5047-5052.

2. Yehuda et al. The incidence of malignancy in clinically benign cystic lesions of the lateral neck: our experience and proposed diagnostic algorithm. Eur Arch Otorhinolaryngology. March 2018.

3. Grønlund et al. The true malignancy rate in 135 patients with preoperative diagnosis of a lateral neck cyst. Laryngoscope investigative otolaryngology. 2016.

4. Stefanickaet al. Incidence and clinical predictors of cystic squamous cell carcinoma metastases in lateral cervical cysts. J LaryngolOtol 2019;1-6

5. P. J. Andrews\& C. E. B. Giddings. Management of lateral cystic swellings of the neck, in the over 40s' age group. The Journal of Laryngology \& Otology. 2003, Vol. 117, pp. 318-320.

6. Regauer et al. Cystic lymph node metastases of squamous cell carcinoma of Waldeyer's ring origin. British Journal of Cancer (1999) 79(9/10), 1437-1442

7.Koch et al. Cystic masses of the lateral neck - Proposition of an algorithm for increased treatment efficiency. Journal of Craniomaxillofacial Surg. Sept 2018.

\section{Hosted file}


Figures.docx available at https://authorea.com/users/359946/articles/481704-1ateral-neckcysts-age-distribution-and-diagnostic-pathway 